\title{
Research and Implements of Learning Process Model Oriented Workflow
}

\author{
Linglin $\mathrm{Li}$ \\ School of Traffic Information \\ Hunan Communication Polytechnic \\ Changsha,Hunan Province China \\ tjptjp@21cn.com
}

\author{
Jinpeng Tang \\ School of Traffic Information \\ Hunan Communication Polytechnic \\ Changsha,Hunan Province China \\ spiderpong@163.com
}

\begin{abstract}
In order to integrate e-learning into the existing work flow management system, we proposed a learning process model oriented workflow. This model is based on WfMC (Workflow Management Coalition) Xml Process Description Language-XPDL standard, combining IMS Learning design specification. The model can either keep feasible route structure of XPDL, or describe learning activity completely. So it is suitable for modeling learning process.
\end{abstract}

Keywords- E-learning; Learning Design; Workflow; Learning Process; Xml Process Description Language

\section{INTRODUCTION}

Over the past decade, with extensive use of E-learning, peoples more and more pay attention to a common learning process Meta model [1]. Merrill theory of teaching affairs, EML Educational Modeling Language and Learning Design specification are research results about the learning process of formal modeling.

IMS's Learning Design specification [2] (Learning Design; LD) based on Netherlands open university Education Modeling Language (EML), focus on learning activities, and it provide a description of learners in the learning environment in a certain order to achieve the intended learning activities, learning objectives. LD can not only simulate the roles of teaching and learning process, but also support the individualized learning process and the large number of online teaching model applications.

Workflow is a kind of fully or partially automate business processes, which according to a set of rules, so that documents, information or tasks can be transferred and implemented between different actors and executors [3]. The workflow technology is imported into e-Learning system so that learning processes are management, monitoring and scheduling executed. Nowadays workflowbased online learning (Workflow-Based E-Learning) is becoming a new generation learning technology [4] [5].

Workflow model is the basis of the application of workflow technology, Workflow Management Coalition (WfMC) released XML-based process definition language (XPDL: Xml based Process Description Language) standardin 2008 [6]. However, the management of the learning process needs to track the learning process, implementation and monitoring. This requires determining the semantic specification of a learning process and provides mechanisms to records capture, store and analyze the learning process. To take advantage of workflow technology to implement and coordinate the learning activities of e-Learning, a semantic model of the learning process must to be established formally. WfMC is the abstraction of business processes; it does not involve learning field. If workflow integrate into e-Learning environment, expansion of e-Learning environment is necessary for stage in the workflow modeling [7].

Based on the above analysis, Based on Workflow Management Coalition's XPDL standards proposed by WfMC and IMS LD specification, this paper proposes a learning process model. Learning process use XPDL, retained the basis interactive elements, combined with IMS LD some elements of learning areas, while maintaining the original XPDL flexible routing architecture, but also completely descript of learning activities. The new model is suit for $g$ process for the learning process modeling.

\section{LEARNING PROCESS MODEL}

This learning process will be defined as: automatically or semi-automatic implementation of the learning process. The management of the learning process is accord learner's knowledge level, learning preferences and learning goals to organize and manage related learning activities. In the learning process, there are two flows. One is data flows, including from one activity to another activity of learningrelated changes in the data and learning environment and knowledge points changes based on different instructional design; the other is the control flows, including the conditions of activating learning activities.

A learning process model's top-level entity is learning unit. A learning unit is the learning process to accomplish a series of learning tasks, such as a chapter or a certain stage of learning to learn, the granularity is common controlled by instructors.

\section{A. Learning Unit}

Learning unit as a container is composed of a group learning process and the relevant data. The relevant data is global, and can be applied to the learning processes of the unit (therefore, these data define only once). Lower in the learning unit defines the learning process, teaching participants, and the relevant data of learning process, learning content, learning environment and other entities, service tools. The core of these is learning processes. And the core of the learning process is learning activities. A learning unit model contains the following entity types: 
- $\quad$ learning process

- learning participant

- relevant data

- learning content

- $\quad$ service tools

Because of these, such as learning environment, the relevant data, learning participants, learning content, service tools entities in the learning process will also be cited, the scope of their role is different and the details will be unified in the learning process.

\section{B. Learning Procesess and Iinternal Entities}

We defined the learning process that is the sum of the learning activities that based on a certain teaching, and in a particular environment (such as specific learning objectives, pre-learning condition) particular users participate (such as learners and instructors).

A learning process is composed of entities, such as learning participants, transition information, activities, learning environment, learning objectives, relevant data, pre-study conditions. Each entity is made up of a number of sub entity elements. Conceptual model of the learning process is shown in Fig. 1.

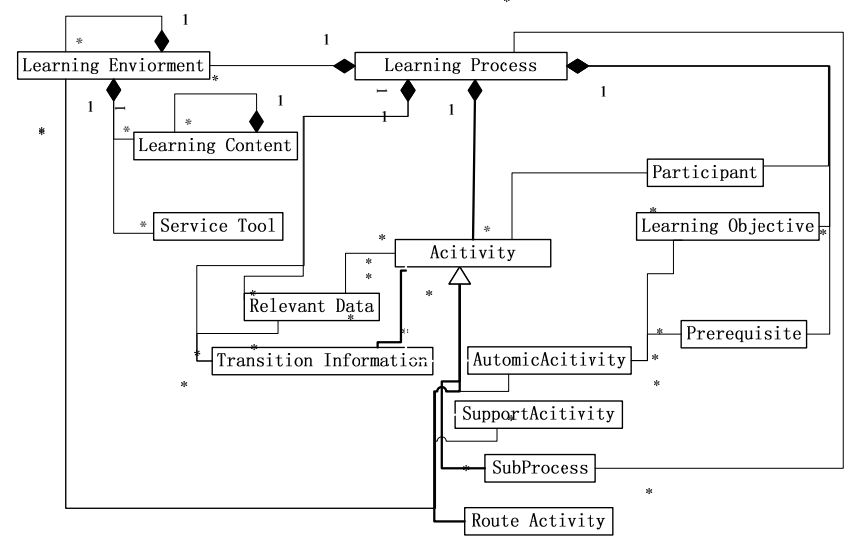

Figure 1 Conceptual Model of Learning Process

The concept of a learning process can be a common description of the following: In order to complete the learning objectives, learning participants according to the learning path to implement a series of activities. These activities include atomic learning activities, routing activities, child learning and support activities. A Learning process uses the transition information and transition constraints to achieve routing constructor, such as sequence, parallel, choice, loop, etc. A learning process involved in activities, the sequence of activities, required data (relevant data) and resources (learning environment).

1) Activity

A learning process is composed of activities. A learning activity is a logical, self-contained task units. Activities related to the description of the activity itself, the activity participants, related data used in activity, the implementation of activities and the end mode. An activity in the learning process is the common abstract from atomic learning activities, routing activities, support activities and child learning process.

Atomic activities: inherited from the element of activity. In addition to the information of activities, the definition of an atomic learning activity related to learning objectives, pre-conditions for learning, learning environment. An atom activity can be described as follow: in order to complete certain learning goals a participant, with a pre- learning condition, based on the selected learning environment to complete learning tasks.

Support activities: support activities is optional, it is used to define collaborative learning. It associates to the supported participants, to support the participants as the roles. Teachers are usually play roles of supporter, but in some learning scenarios, learners also support other learners, or some teachers support other teachers. Compared with the learning activities, support activities need not for the learning objectives, pre-conditions for learning.

Routing activities do not have participants do not need to be put into a specific learning environment, and routing activities running have no effect on workflow relevant data .In essence, it is a virtual activity; it is only responsible for arranging the path of learning activities. Although the transition activity can achieve the path chosen, the use of routing activities can achieve the transfer condition which can not be expressed in the transition activity. With the routing activities, users can model without writing complex conditional expression in the transition activity. Sometimes we need multiple routing activities to describe complex transition. For example:

Combination of XOR and AND split conditions on outgoing transitions from an activity.

Combination of XOR and AND join conditions on incoming transitions to an activity

4) (SubProcess): child learning process .To enhance the learning process of the multiplexing degree, the sub process can refer to external learning processes.

2). Participant

In both LD and XPDL has a similar entity. There participants were divided into two roles: learners and instructors. Different users can play different roles, according to the different permissions for each participant to perform different activities in different learning environments.

3). Learning Environment

Learning environment defines the context in which learning activities. Including resources and reference resources learning activities need. An environmental element consists of three basic entities: learning content, service tools, sub-environment. Learning content is any entity used in the learning process: such as HTML, text, video, animation, audio and so on. Service tools are the applications used in the learning process. Service tools may be integrated into the learning management system; it could also be a reference to external applications.

4) Service Tool: In this learning process model service tools in corresponding XPDL the application entities. A service to can be referenced through its unique identifier in 
the learning environment, and learning activities is running in the specified learning environment, and that it is associated with the learning environment. Learning services are performed by the service tools, but only the type of learning services is specified during the process of learning process modeling; after the learning process model is instantiated, the user specify service tools to execute learning activities. The service tool is reference by its URL. In the learning process model, we define parameters set through that support a more flexible use of service tools.

5) Learning content: In this, we defined learning content as digital and non-digital learning resources required to achieve learning activities. IMS-LOM models can refer to the definition of learning content. Learning Objects in LOM specification are classified, including exercises, simulations, questionnaires, graphics, exercises, tests, lectures, etc.; for compatibility with existing and future metadata standards customize metadata can also be used.

6) Learning Objective

Learning objectives describe the goals after the learner accomplish a series of learning activities to achieve. Learning process modelers can describe the objectives from two levels: global learning objectives from the higher layer of the learning process, the individual learning objective of a learning activity from the lower layer of learning activities.

7) Pre-conditions for learning (Prerequisite)

Prerequisite define the knowledge learner have to familiar with before they begin a learning activity.

8) Transition Information

In LD complex process are modeled by conditions and notify, it often covered multiple acts or methods, so it too complex to common user [8]. In this paper, we describe the activity sequence by transition information, which includes conditions of the activities of the transfer, and some of the more complex control constraints. Activity network edges can be marked on the transfer conditions. When the conditions are met metastasis; if there is no marked transfer condition, the control of these transfers need to use more control constraints and conditions, we can define entities in the learning activities description. For examples: input transfer described by JOIN, the transfer constraints described using AND or XOR; for output transfer with SPLIT; the constraints on the outflow transfer described using AND or XOR. The combination of transition information and routing activities can be used to describe any complexity learning process.

9) Relevant Data

Learning process relevant data are those in the learning process, each process instance needs to use or create the data. Learning activities must use these data in the implementation, which can also be used to deliver intermediate results or persistent message of learning activities. In the transfer information condition expressions also need these data. For example, learning activities accord the previous learning activity to choose the next learning activities to be performed, and the learning results may serve as a kind of relevant data transferring between the learning activities.

\section{LEARNING PROCESS DESCRIPTION LANGUAGE}

In the conceptual model, we described the constituent entities of the learning process mode and the relationships between them, then use the schema to accurately define the various entities and their relations, which we call learning process description language (LPDL). Users can set up the learning process model based on LPDL. The final results of the learning process model are the xml document. The following is the key elements of learning process in the schema information model.

\section{A. Learning Process}

Elements defined in the learning process are shown in Fig. 2, the sub-elements described as follow:

Name

ProcessHeader Element set described the characteristics of learning processes

TypeDeclarations Data type list used in learning processes LearningParticipants Participants list in learning process

DataFields Relevant data list in learning processes

ExtendedAttributes Extended property list

LearningObjectives Learning objectives List in learning processes

Prerequisites Pre-learning conditions list required to start a learning process

Activities Learning activities list in learning processes

Transitions Transition information list in learning processes

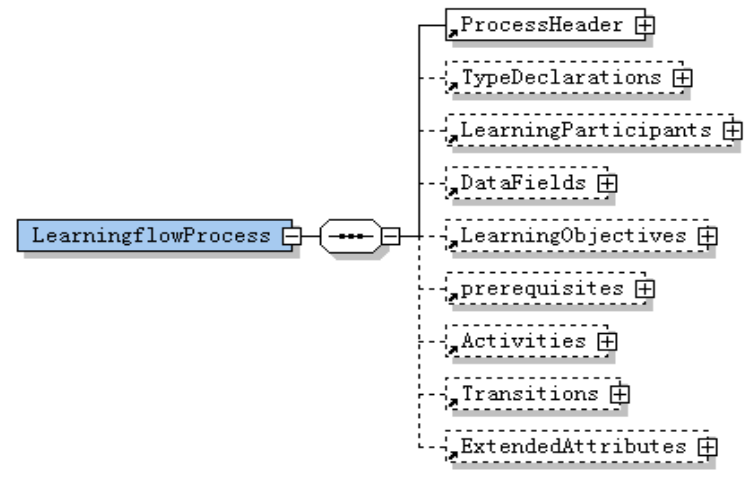

Figure 2 elements of the learning process

\section{B. Activity}

Elements defined in the Activity are shown in Fig. 3, the sub-elements described as follow:

Name

Description

Description

ActivityType descriptions of activities

types of activities

LearningParticipants learning activities participants

ExtendedAttributes extended property list

TransitionRestrictions transfer restriction list of activities 


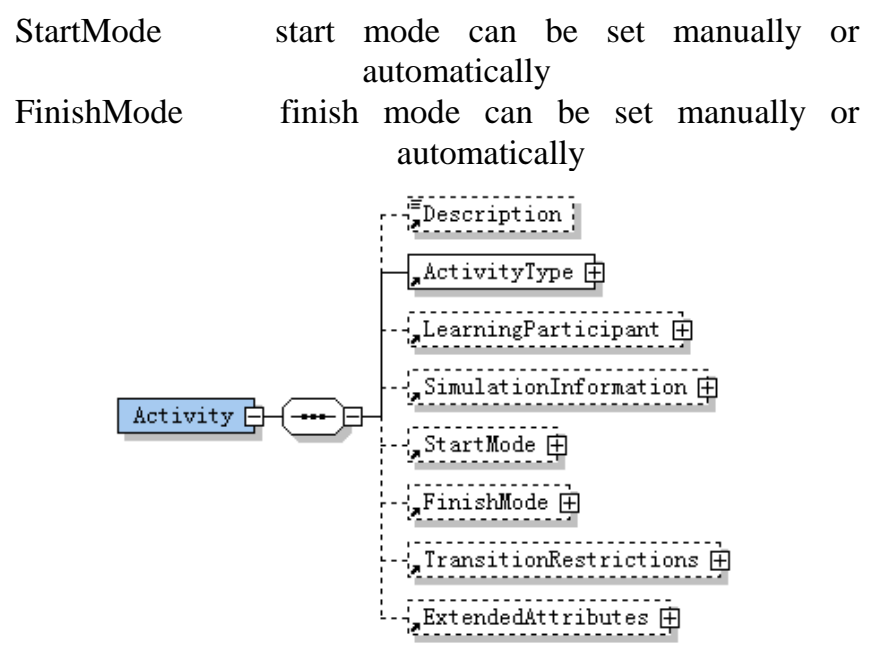

Figure 3 Elements of the activities

\section{LPDL Map to XPDL}

Based on the e-learning integrated into the workflow management system, it is necessary to take the LPDL learning process model to run in WfMC management system. To do this requires LPDL need to be fully mapped to XPDL. Since the LPDL proposed is based on the dynamic elements of XPDL, so long as the static elements can be mapped to XPDL.

Concluding from the above analysis, LPDL are compatible with the workflow management systems based on XPDL standard. That means the LPDL learning process model can be integrated into existing workflow management system.

\section{CONCLUSION}

Integrated workflow model and learning activities model, a learning process model is proposed. This model combined WfMC's XPDL standards and IMS's LD specification. It is based on XPDL, combined with some of the static elements of IMS LD, extended XPDL standard, to enable a complete description of learning processes, and it is suitable for modeling in s learning areas. At the same time the paper use static entity of IMS LD to extend XPDL, therefore LFDL complete mapping to XPDL is feasible, that is LFDL is compatible with XPDL standard. So at the flow describing level e-learning integrated into workflow is realized.

\section{ACKNOWLEDGMENT}

The research was supported by Scientific Research Foundations of Hunan Provincial Education Department (Project No.09C598).

\section{REFERENCES}

[1] Hummel Hans,Koper.R,Tattersall.Colin,”LO->LA: From a Learning Object Centric View Towards A Learning Activity Perspective,"Technology, Instruction, Cognition and Learning,vol.12,pp.211-274,March 2006.
[2] IMS.LearningDesign Specification, retrieved August 16, 2010 from /http://www.imsglobal.org/learningdesign/index.html.

[3] W.M.P. van der Aalst,A.H.M. ter Hofstede,B Kiepuszewski,”Advanced Workflow Patterns,” Distributed and Parallel Databases,Vol.14,No.1,pp.5-11,January 2003.

[4] Yong, Jianming .”workflow-based E-learning Platform,"Proc. 9th International Conference on Computer Supported Cooperative Work in Design, May 2005,pp. 1002-1007.

[5] Koper, R., "Current Research in Learning Design,” Journal of Educational Technology \& Society, International Forum of Educational Technology \& Society,Vol. 9,No.1, 2006, pp. 13-22

[6] WfMC.Workflow Process Definition Interface-XML Process Definition Language (XPDL) [S].WFMC-TC-1025-Oct-10-08A,2008

[7] Miao, Y., Hoeksema, K., Hoppe, H.U. and Harrer, A., "CSCL Scripts: Modelling Features and Potential Use,"Proc. Proceedings of th 2005 conference on Computer support for collaborative learning: learning 2005: the next 10 years!, International Society of the Learning Sciences Press,June.2005,pp. 423-432.

[8] Caeiro M, Anido L, Llamas M,”A Critical Analysis of IMS Learning Design,” Proc.Fourth IEEE International Conference on Advanced Learning Technologies (ICALT'04) , IEEE Press ,March 2003,pp.363-367. 